\title{
Ongoing outbreak of human adenovirus-associat- ed acute respiratory illness in the Republic of Korea military, 2013 to 2018
}

Jae-Hoon $\mathrm{Ko}^{1,2,}{ }^{,}$, Hyeong-taek Woo ${ }^{3,}{ }^{*}$, Hong Sang $\mathrm{Oh}^{1}$, Song Mi Moon ${ }^{1,4}$, Joon Young Choi ${ }^{5}$, Jeong Uk Lim Donghoon Kim ${ }^{3}$, Junsu Byun 3 , Soon-Hwan Kwon ${ }^{6}$, Daeyoun $\mathrm{Kang}^{6}$, Jung Yeon $\mathrm{Heo}^{7}$, and Kyong Ran Peck ${ }^{2}$

${ }^{1}$ Division of Infectious Diseases, Department of Internal Medicine, Armed Forces Capital Hospital, Seongnam; ${ }^{2}$ Division of Infectious Diseases, Department of Medicine, Samsung Medical Center, Sungkyunkwan University School of Medicine, Seoul; ${ }^{3}$ Department of Preventive Medicine, Armed Forces Medical Command, Seongnam; ${ }^{4}$ Division of Infectious Diseases, Department of Internal Medicine, Seoul National University Bundang Hospital, Seongnam; ${ }^{5}$ Division of Pulmonary and Critical Care Medicine, Department of Internal Medicine, Armed Forces Capital Hospital, Seongnam; ${ }^{6}$ Department of Infectious Diseases, Research Center of Infectious and Environmental Diseases, Armed Forces Medical Research Institute, Daejeon; ${ }^{7}$ Department of Infectious Diseases, Ajou University School of Medicine, Suwon, Korea
Background/Aims: Human adenovirus type 55 (HAdV-55), an emerging epidemic strain, has caused several large outbreaks in the Korean military since 2014, and HAdV-associated acute respiratory illness (HAdV-ARI) has been continuously reported thereafter.

Methods: To evaluate the epidemiologic characteristics of HAdV-ARI in the Korean military, we analyzed respiratory virus polymerase chain reaction (RV-PCR) results, pneumonia surveillance results, and severe HAdV cases from all 14 Korean military hospitals from January 2013 to May 2018 and compared these data with nationwide RV surveillance data for the civilian population.

Results: A total of 14,630 RV-PCRs was performed at military hospitals. HAdV (45.4\%) was the most frequently detected RV, followed by human rhinovirus (12.3\%) and influenza virus (6.3\%). The percentage of the military positive for HAdV was significantly greater than the percentage of civilians positive for HAdV throughout the study period, with a large outbreak occurring during the winter to spring of 2014 to 2015 . The outbreak continued until the end of the study, and non-seasonal detections increased over time. The reported number of pneumonia patients also increased during the outbreak. Case fatality rate was $0.075 \%$ overall but $15.6 \%$ in patients with respiratory failure. The proportion of severe patients did not change significantly during the study period.

Conclusions: A large HAdV outbreak is currently ongoing in the Korean military, with a trend away from seasonality, and HAdV-55 is likely the predominant strain. Persistent efforts to control the outbreak, HAdV type-specific surveillance, and vaccine development are required.

Keywords: Adenoviridae; Disease outbreaks; Korea; Military personnel; Type 55

\section{Correspondence to Kyong Ran Peck, M.D.}

Division of Infectious Diseases, Department of Medicine, Samsung Medical Center, Sungkyunkwan University School of Medicine, 81 Irwon-ro, Gangnam-gu, Seoul o6351, Korea Tel: +82-2-3410-0329, Fax: +82-2-3410-0064, E-mail: krpeck@skku.edu

https://orcid.org/0000-0002-7464-9780

*These authors contributed equally to this work.
Received: March 18, 2019

Revised : May 1, 2019

Accepted: July 25, 2019 


\section{INTRODUCTION}

Acute respiratory illness (ARI) associated with human adenovirus (HAdV) usually occurs in infants and children [1], with severe cases reported sporadically in adults [2]. Outbreaks of HAdV-associated ARI (HAdVARI) in adults have been reported in communal living populations, including school, hospital, and military populations [3-8]. HAdV-55, a novel recombinant strain of HAdV-11 and HAdV-14 that causes ARI in adults [9], emerged as an epidemic strain in Asian and European countries from 2004 [6,8,10-18]. In the Republic of Korea, fatal cases of HAdV-55 pneumonia among military personnel have been reported since 2012 [19], and a large military outbreak associated with HAdV-55 was observed in the winter of 2014 [20]. Although several outbreak studies at different sites have been conducted [21-24], epidemiologic evaluation of the entire military population has yet to be conducted. To assess the current status of the HAdV outbreak, we designed an epidemiologic study for all 14 military hospitals in the Republic of Korea and compared these data to nationwide surveillance data for the Korean civilian population.

\section{METHODS}

\section{Study design and data collection}

For nationwide epidemiologic investigation, we reviewed respiratory virus polymerase chain reaction (RV-PCR) results of all 14 military hospitals (Hospitals A to N) affiliated with the Korean Armed Forces Medical Command (AFMC) during the period of January 2013 to May 2018. The 14 military hospitals with a total of 4,710 beds located throughout the nation cover patients from neighboring troops, while Hospital J acts as a referral center for the military hospitals. Geographic regions and number of beds in each hospital are presented in Supplementary Table 1. RV-PCR was introduced to military hospitals in 2011 [25] and became available at all 14 hospitals in 2013. RV-PCR was selectively performed initially [25], but since the 2014 to 2015 outbreak of HAdV55 has been strongly recommended as a routine test for all ARI patients with persisting fever or pneumonia [20]. Oral vaccine for HAdV-4 and -7 was not supplied to military personnel during the study period. Collected data

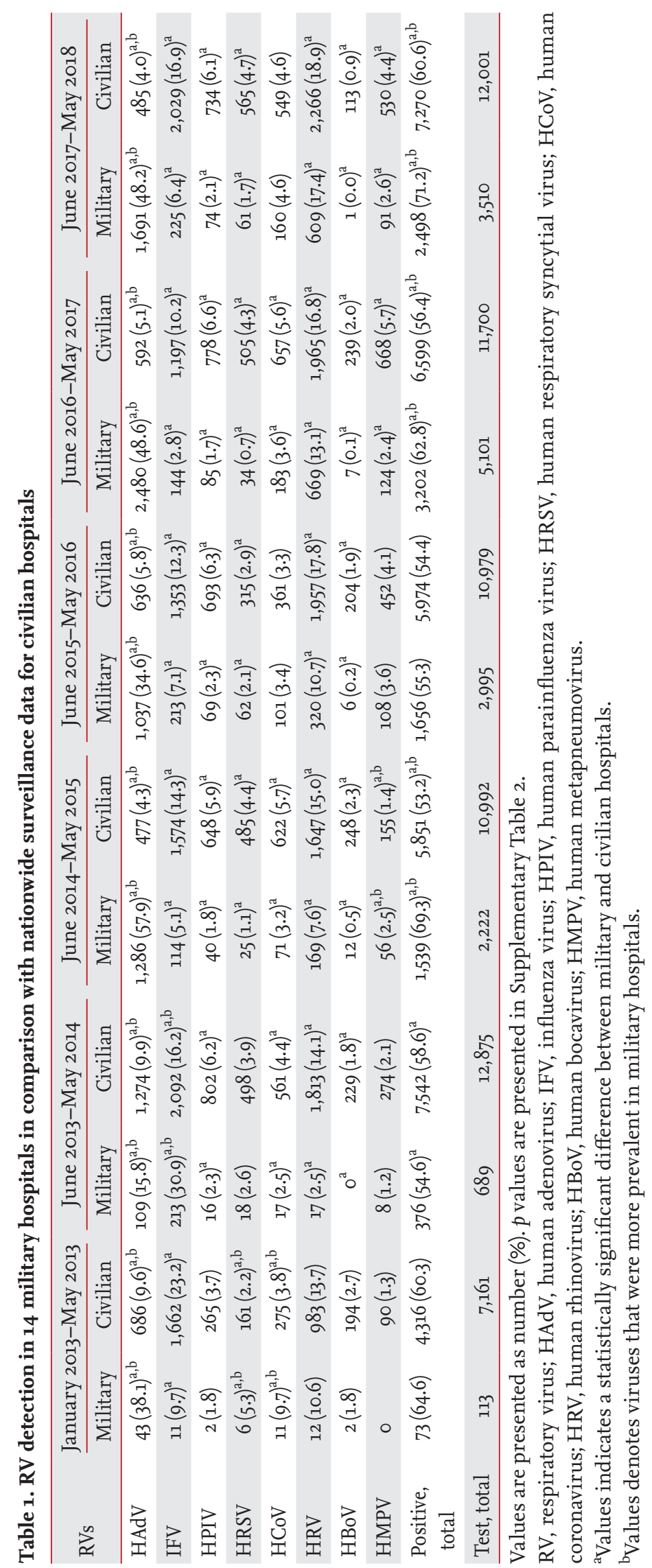


included the date and hospital of collection, affiliation of the patient, and RV-PCR results. We could not review electronic medical records (EMRs) of the entire patient population due to the large size of the target population; therefore, information about specimen type (throat swab or sputum) and type of illness (upper or lower respiratory tract infection) was not collected.

To compare the epidemiology of the military with that of the civilian population, we used data from a nationwide RV surveillance system administered by the Korea Centers for Disease Control and Prevention (KCDC) that is reported weekly on the KCDC website (http://www. cdc.go.kr) [1]. RV surveillance findings for new recruits were used for comparison. Since August 2015, the RV status of military personnel at the time of enrollment has been assessed by the Korea Army Training Center for surveillance purposes. Symptomatic new recruits are tested for RV-PCR within one week of entrance (before initiation of major training programs).

To investigate severe HAdV-ARI cases and determine the overall number of pneumonia patients, medical surveillance data reported to the AFMC were used. Severe HAdV-ARI cases were defined as those that required cidofovir treatment, mechanical ventilation (MV) support, or extracorporeal membrane oxygenation (ECMO). EMRs of these patients were reviewed retrospectively. The number of pneumonia patients at all 14 military hospitals is reported weekly to the AFMC according to the International Classification of Disease and Related Health Problems, 1oth revision (ICD-10, codes J12-J18). This study was approved by the Institutional Review Board of the AFMC (AFMC-18-IRB-051) and informed consent was waived by the board.

\section{Laboratory tests}

During the study period, PCR analyses for eight RVs (HAdV, influenza virus [IFV], human parainfluenza virus [HPIV], human respiratory syncytial virus [HRSV], human coronavirus [HCoV], human rhinovirus [HRV], human bocavirus [HBoV], and human metapneumovirus [HMPV]) were performed at three clinical laboratories depending on period and hospital (details are presented in the Supplementary Material).

\section{Review of HAdV molecular typing data}

As RV-PCR tests do not include molecular typing of HAdV, we made an effort to collect all available HAdV-typing data for the Korean military. To review published data, we searched PubMed up to October 2018 using the following search protocol: ((Adenovirus OR Adenoviral OR HAdV) AND Korea), or ((Adenovirus or Adenoviral OR HAdV) AND 55). For ongoing studies and unpublished data, we contacted all investigators who obtained approval from the two existing Institutional Review Boards of the AFMC and Armed Forces Capital Hospital for HAdV-related studies. Information collected about HAdV-typing is presented in the Discussion.

\section{Statistical analysis}

Positive percentages of each RV and epidemiologic characteristics of HAdV and other RVs were compared between military and civilian hospitals using chi-square and Fisher's exact tests. Student's $t$ test was used to compare seasonal variations. To evaluate if there were increasing or decreasing trends, a linear regression model was used. All $p$ values were two-tailed, and those that were $<0.05$ were considered statistically significant. IBM SPSS Statistics version 20.o for Windows (IBM Co., Armonk, NY, USA) was used for all statistical analyses.

\section{RESULTS}

\section{Epidemiology of RV in the Korean military in com- parison with the civilian population}

A total of 14,630 RV-PCRs was performed during the study period at the 14 military hospitals, and 9,344 (63.9\%) of the tests were positive. HAdV was the most frequently detected respiratory virus $(6,646 / 14,630,45.4 \%)$, followed by $\operatorname{HRV}(1,796 / 14,630,12.3 \%)$, IFV (920/14,630, 6.3\%), HCoV (543/14,630, 3.7\%), HMPV (387/14,630, 2.7\%), HPIV (286/14,630, 2.0\%), HRSV (206/14,630, 1.4\%), and HBoV (28/14,630, $0.2 \%)$. In the same period, civilian hospitals performed 65,780 RV-PCRs, 37,552 of which were positive $(57.1 \%)$. HRV was the most commonly identified respiratory virus $(10,631 / 65,780,16.2 \%)$, followed by IFV (9,970/65,780, 15.2\%), HAdV (4,149/65,780, 6.3\%), HPIV (3,920/65,780, 6.0\%), HCoV $(3,024 / 65,780,4.6 \%)$, HRSV $(2,529 / 65,780,3.8 \%)$, HMPV $(2,168 / 65,780,3.3 \%)$, and $\mathrm{HBoV}(1,125 / 65,780,1.7 \%)$.

To determine overall epidemiologic trends in RVs, 
the positive percentages of each RV in the military per month and the number of cases detected were compared with those of civilian hospitals (Fig. 1). Among the eight RVs, only HAdV exhibited a markedly higher positivity in the military than in civilian hospitals. In contrast to the civilian data that did not show any seasonal varia- tion in HAdV, HAdV detection in the military surged in the winter and spring of the 2014 to 2015 period, with a more than four-fold increase compared to the nadir of the summer to fall of 2015 (Supplementary Table 2). Peak detection during this period was in March. The average HAdV-positive percentages were also significantly high-

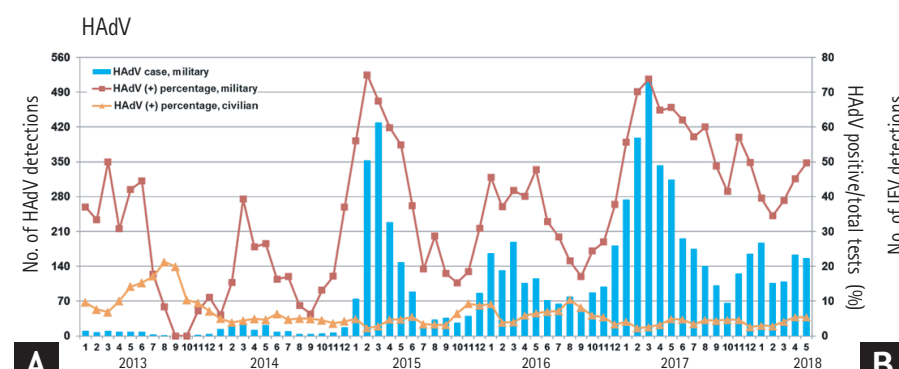

A

HPIV
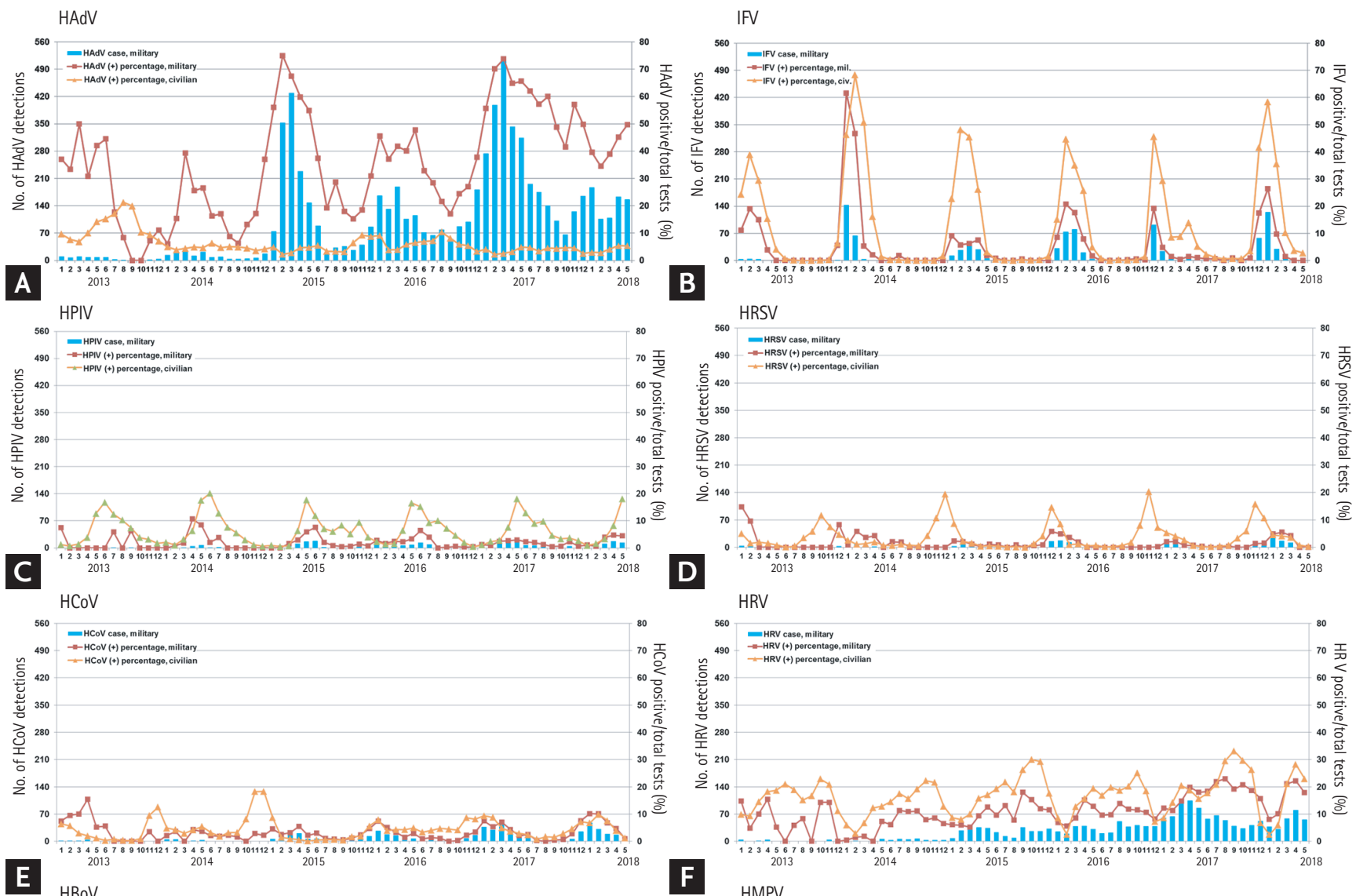

B
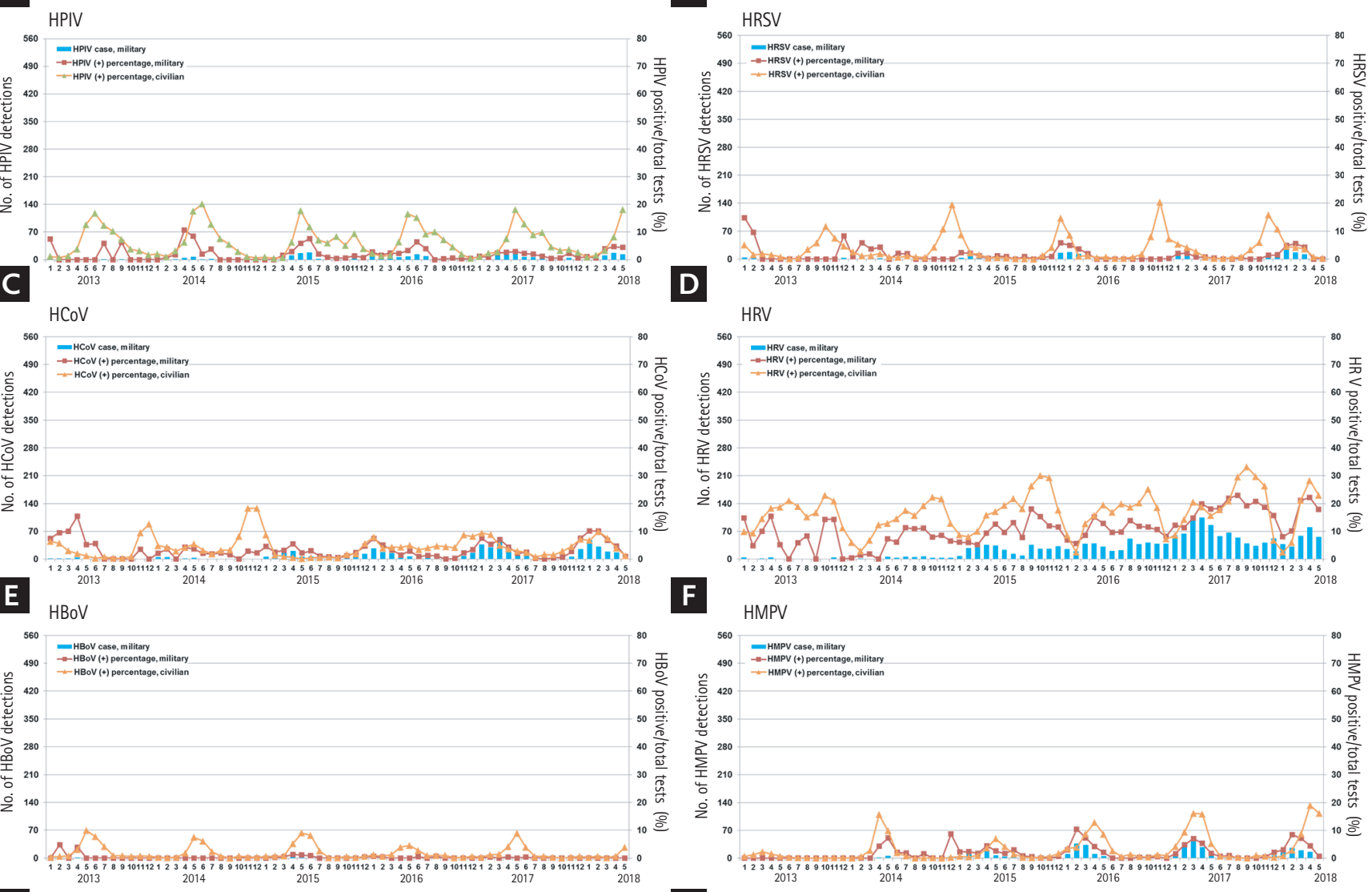

G
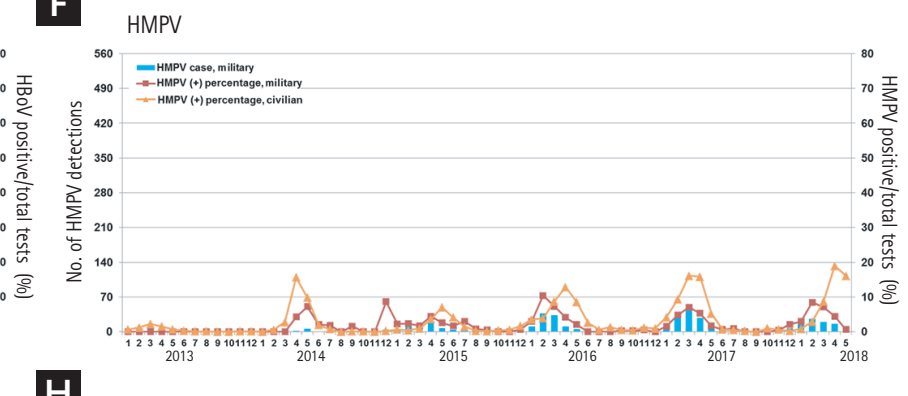

$\mathbf{H}$

Figure 1. Epidemiologic trends of eight respiratory viruses in the Korean military in comparison with the civilian population. Respiratory virus polymerase chain reaction results of 14 military hospitals from January 2013 to May 2018 are presented in addition nationwide surveillance data from civilian hospitals. From the winter to spring seasons of 2014 to 2015 , a large human adenovirus (HAdV) outbreak occurred in the military and persisted thereafter. Previous studies and unpublished data suggest that this ongoing outbreak is associated with HAdV-55. (A) HAdV, (B) influenza virus (IFV), (C) human parainfluenza virus (HPIV), (D) human respiratory syncytial virus (HRSV), (E) human coronavirus (HCoV), (F) human rhinovirus (HRV), (G) human bocavirus (HBoV), and (H) human metapneumovirus (HMPV). 


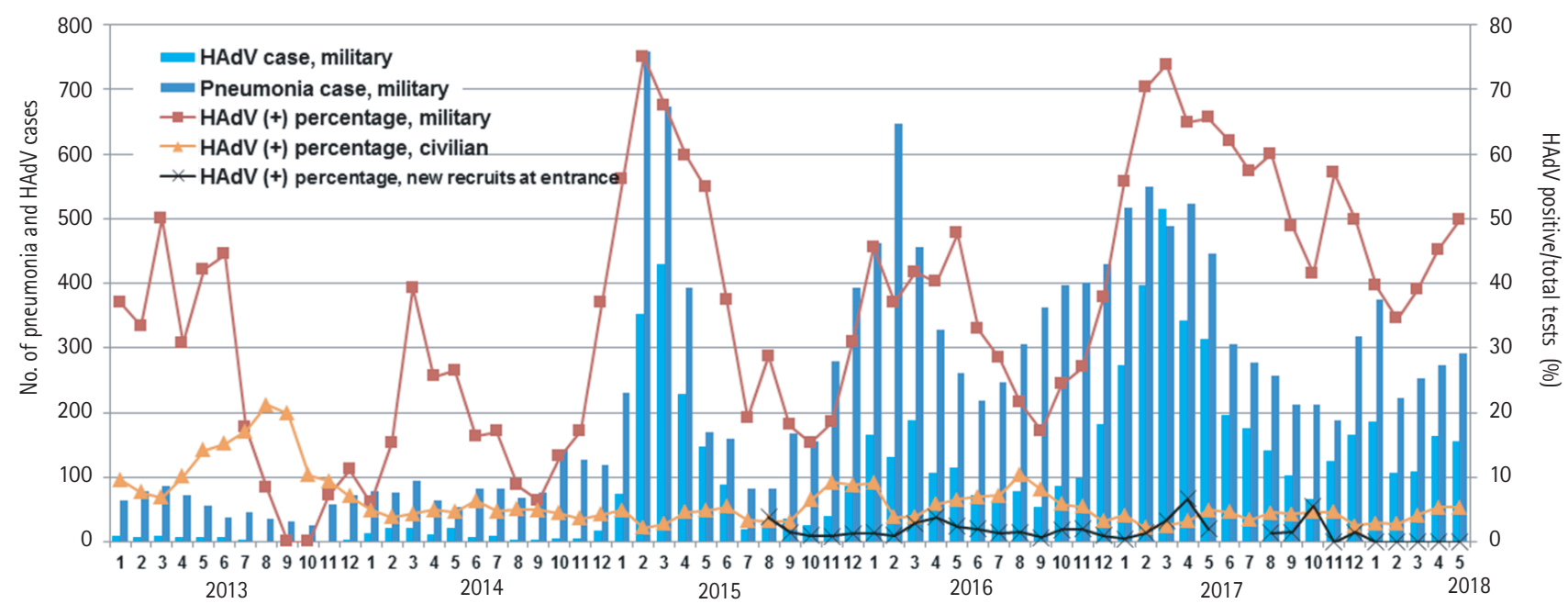

Figure 2. Pneumonia surveillance and human adenovirus (HAdV) detections in new recruits at the time of entrance as well as HAdV detections in military and civilian hospitals. Reported pneumonia cases increased over time with the ongoing HAdV outbreak in the military, while HAdV detection proportions in new recruits at the time of entrance were consistently low.

er in the winter to spring period than the summer to fall period (58.3\% vs. $22.8 \%, p<0.001)$. However, this seasonal variation gradually weakened with increasing HAdV detection during the summer and fall seasons of 2016 . In the 2016 to 2017 period, the average HAdV positivity value was not statistically different between the winter to spring period and summer to fall period (61.3\% vs. $54.4 \%, p=0.295)$.

For statistical comparison of military and civilian populations, we documented number of PCR-positive RV cases from June of each year to May of the following year to include HAdV outbreak season (Table 1 and Supplementary Table 3). For the entire evaluation period, positive percentage of HAdV was significantly higher in military than civilian hospitals (all $p<0.05$ ). Seasonal variations were clearly observed in the other seven RVs in civilian hospitals, as illustrated in the scale-adjusted graph shown in Supplementary Fig. 1. A similar seasonality was noticed in the military starting in 2014, with increasing numbers of RV-PCR tests. Epidemiologic patterns of each RV are described in the Supplementary Material.

\section{Evaluation of ongoing HAdV outbreak in the Korean military \\ Data from pneumonia surveillance according to ICD-10 codes and HAdV detection in new recruits at the time}

of entrance are presented with HAdV detections in military and civilian hospitals (Fig. 2). During the study period, a total of 15,503 pneumonia patients was reported to the AFMC. The number of pneumonia patients increased markedly in the 2014 to 2015 period $(2,922$ patients compared to 673 patients in the previous period) (Supplementary Table 4), with the same seasonal variation in HAdV. Although there was an outbreak of IFV during the 2013 to 2014 period, the number of pneumonia patients did not increase accordingly. Overall, number of new recruits positive for HAdV at the time of entrance (average of 1.8\%) was less than the positive proportions among civilians (average of $4.9 \%$ ). HAdV detection in new recruits at entrance did not exhibit seasonal variation.

\section{Proportion of severe HAdV-ARIs and clinical courses of five fatal cases}

During the study period, 106 cases were treated with cidofovir (1.6\% of 6,646 HAdV cases), 32 cases progressed to respiratory failure requiring MV support (0.5\%), and 15 cases received ECMO support (0.2\%) (Supplementary Table 5). A total of five fatal cases was reported, with a case fatality rate of $0.075 \%$ for overall HAdV-ARIs. The case fatality rate was $15.6 \%$ among patients who progressed to respiratory failure. The proportion of severe patient s did not change significantly during the study period 


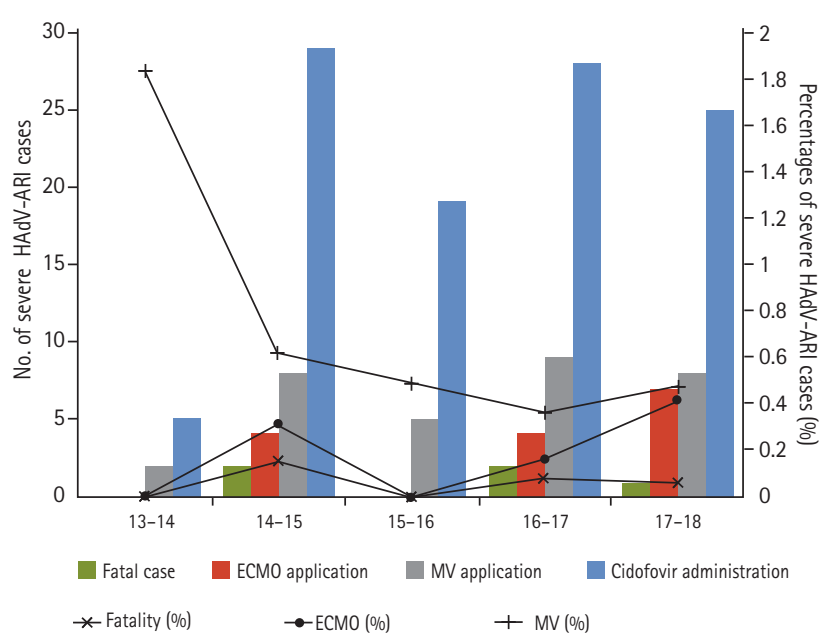

Figure 3. Numbers and proportions of severe human adenovirus acute respiratory illness (HAdV-ARI) cases in the Korean military. Severe HAdV-ARI included those cases treated with cidofovir, those that required mechanical ventilation (MV) or extracorporeal membrane oxygenation (ECMO) support, and those that died.

(Fig. 3). Clinical courses of the five fatal HAdV infections are presented in Table 2. All patients were males in their early 20 s with a low military rank. There was a median of 6 days from symptom onset to respiratory failure. Cidofovir was administered in three patients within 2 days after progression to respiratory failure. ECMO was applied in three patients. Patients D and E received ECMO support on the same day of respiratory failure, implying a rapidly progressive disease. Median survival was 18 days and ranged from 12 to 66 days. Causal relationships of HAdV-ARI and death are presented in the footnote of Table 2.

\section{DISCUSSION}

The first epidemiologic study of RVs in the Korean military in 2006 reported a predominance of HAdV-7 [26], which was associated with a large HAdV outbreak in Korean children in 1996 and subsequent small outbreaks [27]. Although detection of HAdV-7 was as high as $61.0 \%$, severe HAdV-ARI cases were not reported [26,28]. From January 2011 to March 2013, six cases of severe adenoviral pneumonia were identified with 50\% mortality, while such severe adenoviral pneumonia was not reported previously in the Korean military [25,28]. An epi-

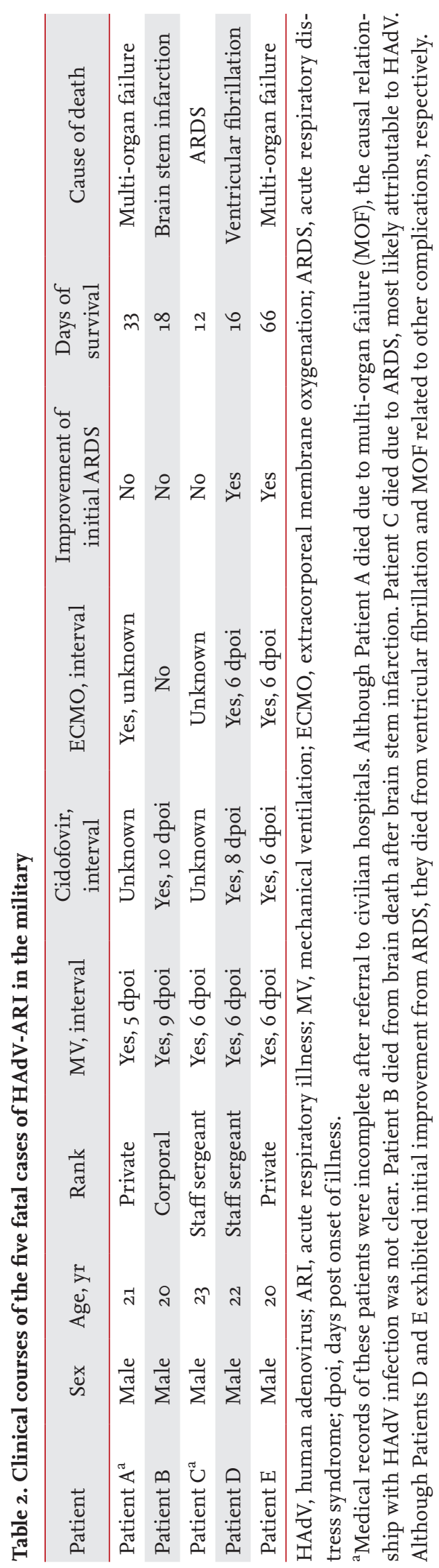

https://doi.org/10.3904/kjim.2019.092 
demiologic study based on HAdV molecular typing was conducted from January 2013 to April 2014, and HAdV$55(42.0 \%)$ was the most frequently identified strain, followed by HAdV-4 (13.0\%), HAdV-5 (1.4\%), and HAdV-6 (1.4\%) [23]. Severe HAdV-ARIs requiring MV support were exclusively due to HAdV-55 in that period $[19,23]$. From the winter to spring seasons of 2014 to 2015, a large HAdV outbreak occurred in the military and persisted into the next season [20]. Molecular typing of HAdV was performed for 74 samples collected in 2016; all were identified as HAdV-55 [20]. In other unpublished data from ongoing studies in the military, HAdV-55 was strongly predominant during the 2016 to 2017 and 2017 to 2018 periods. According to a surveillance study evaluating 1,079 ARI cases in four military training centers from the 37th week of 2016 to the 36th week of 2018,509 of $518 \mathrm{HAdV}$-ARIs (98.3\%) were caused by HAdV-55, and HAdV-2 was detected in nine cases $(1.8 \%$, data acquired by personal communication with J.Y.H.). In a cytokine study of $23 \mathrm{HAdV}$ pneumonia patients admitted to Hospital J from September 2017 to June $2018,100 \%$ of cases were due to HAdV-55 (data acquired by personal communication with J.U.L.). The predominance of HAdV55 was also observed in a serologic study evaluating 52 HAdV-ARIs from April 2018 to June $2018 ; 100 \%$ of cases were HAdV-55 positive (data acquired by personal communication with S.H.K.). Although molecular typing of HAdV was not performed in the present study, the abovementioned findings suggest the following plausible scenario: after introduction of HAdV-55 to the Korean military in 2006, it became predominant in the early 2010 s and is currently causing the largest ongoing outbreak that started in winter 2014 .

In addition to epidemicity in the adult population, rapidly progressive and potentially fatal acute respiratory distress syndrome is a key concern of HAdV-55 infection [13]. Many studies have reported severe cases $[6,8,14,15,29,30]$, and large outbreak studies have reported case fatality rates of approximately $0.4 \%[8,20]$. In the present analysis of $6,646 \mathrm{HAdV}$-ARI cases, the overall case fatality rate was $0.075 \%$, less than $0.1 \%$. This can explain why previous outbreaks in China involving fewer than 1,000 patients did not report mortality [14], while sporadic reports of fatality due to HAdV-55 have persisted [13]. Reports of several severe or fatal cases may imply a large outbreak of HAdV-55 in the background community [13]. Although the fatality rate is not as high as for emerging respiratory viruses such as Middle East respiratory syndrome coronavirus (MERS-CoV) [31-33], it is important for public health officials to recognize that a pathogen responsible for the common cold may cause severe and potentially fatal infections.

Of note, case fatality of HAdV patients with respiratory failure was as high as $15.6 \%$. For fatal cases, the median time of MV and ECMO support was 6 days from symptom onset, suggesting a rapidly progressive disease. The times to MV and ECMO support were markedly shorter than those documented for MERS patients (12 and 16 days, respectively) [31]. Although cidofovir was administered in three patients, it was administered after progression to respiratory failure. While early administration of cidofovir has been reported to be associated with favorable outcomes, the chance for early administration is slim in rapidly progressive cases $[2,19,34]$. Because there are few efficacy-proven treatment modalities for HAdV-ARI, control of outbreaks is emphasized to reduce the overall burden of the disease. To control HAdV outbreaks, AFMC has implemented several measures, including emphasis of hand hygiene, use of personal masks, cohort-isolation of trainees with ARI, and early referral of febrile patients to hospitals. Because HAdV outbreaks are more prominent among trainees and low-rank soldiers recently discharged from training centers $[20,21,34]$, such measures have been emphasized in training centers for new recruits [24]. Although an oral vaccine for HAdV-4 and -7 successfully controlled HAdV-ARI outbreaks in the United States military [35], it has not been adopted in the Korean military because HAdV-ARI is known to exhibit type-specific immunity, and HAdV-55 shares immunogenicity with HAdV-14 [9,36,37]. Several multi-valent HAdV vaccines targeting HAdV-55 are currently under development, but clinical application of these vaccines will take time [36,38]. More resources should be focused on vaccine development, and HAdV molecular typing must be implemented in surveillance systems for proper selection of HAdV vaccines in the future.

The present epidemiologic study had several limitations. First, we mainly used reported data of RV-PCR tests without molecular typing. However, the present ongoing HAdV outbreaks are likely primarily due to HAdV-55, considering the previously reported studies, 
seasonal outbreak patterns, and data from ongoing unpublished studies. Second, because we could not review EMRs of all HAdV cases, clinical presentation of HAdV55 infections was based on previous studies [20,22]. Third, as only patients visiting hospitals were evaluated, the proportion of RVs is likely biased. Nevertheless, this bias would have been similar in surveillance studies of civilian hospitals and would not critically affect epidemiologic comparisons between military and civilian hospitals.

In conclusion, a large HAdV outbreak that started in 2014 is currently ongoing in the Korean military, and HAdV-55 is likely the predominant strain. Efforts to control this outbreak should be strengthened, and implementation of HAdV molecular typing for surveillance and vaccine development is required.

\section{KEY MESSAGE}

1. A large human adenovirus (HAdV) outbreak occurred in the Korean military in the winter of 2014 and continued until the end of the study (May 2018).

2. HAdV-55 was likely the predominant strain.

3. Case fatality rate of HAdV-associated acute respiratory illness was $0.075 \%$ overall but $15.6 \%$ in patients with respiratory failure.

\section{Conflict of interest}

No potential conflict of interest relevant to this article was reported.

\section{Acknowledgments}

We thank medical officers of the Armed Forces Medical Research Institute for providing RV surveillance data for new recruits.

This research was supported by a grant(18172MFDS255) from the Ministry of Food and Drug Safety in 2018.

\section{REFERENCES}

1. Kim JM, Jung HD, Cheong HM, et al. Nation-wide surveillance of human acute respiratory virus infections between 2013 and 2015 in Korea. J Med Virol 2018;90:1177-
1183.

2. Hakim FA, Tleyjeh IM. Severe adenovirus pneumonia in immunocompetent adults: a case report and review of the literature. Eur J Clin Microbiol Infect Dis 2008;27:153158.

3. Klinger JR, Sanchez MP, Curtin LA, Durkin M, Matyas B. Multiple cases of life-threatening adenovirus pneumonia in a mental health care center. Am J Respir Crit Care Med 1998;157:645-649.

4. Ryan MA, Gray GC, Smith B, McKeehan JA, Hawksworth AW, Malasig MD. Large epidemic of respiratory illness due to adenovirus types 7 and 3 in healthy young adults. Clin Infect Dis 2002;34:577-582.

5. Top FH Jr, Dudding BA, Russell PK, Buescher EL. Control of respiratory disease in recruits with types 4 and 7 adenovirus vaccines. Am J Epidemiol 1971;94:142-146.

6. Chmielewicz B, Benzler J, Pauli G, Krause G, Bergmann F, Schweiger B. Respiratory disease caused by a species B2 adenovirus in a military camp in Turkey. J Med Virol 2005;77:232-237.

7. Hierholzer JC, Pumarola A, Rodriguez-Torres A, Beltran M. Occurrence of respiratory illness due to an atypical strain of adenovirus type 11 during a large outbreak in Spanish military recruits. Am J Epidemiol 1974;99:434-442.

8. Zhu Z, Zhang Y, Xu S, et al. Outbreak of acute respiratory disease in China caused by B2 species of adenovirus type 11. J Clin Microbiol 2009;47:697-703.

9. Walsh MP, Seto J, Jones MS, Chodosh J, Xu W, Seto D. Computational analysis identifies human adenovirus type 55 as a re-emergent acute respiratory disease pathogen. J Clin Microbiol 2010;48:991-993.

10. Kajon AE, Dickson LM, Metzgar D, Houng HS, Lee V, Tan $\mathrm{BH}$. Outbreak of febrile respiratory illness associated with adenovirus 11a infection in a Singapore military training cAMP. J Clin Microbiol 2010;48:1438-1441.

11. Cao B, Huang GH, Pu ZH, et al. Emergence of community-acquired adenovirus type 55 as a cause of community-onset pneumonia. Chest 2014;145:79-86.

12. Lu QB, Tong YG, Wo Y, et al. Epidemiology of human adenovirus and molecular characterization of human adenovirus 55 in China, 2009-2012. Influenza Other Respir Viruses 2014;8:302-308.

13. Sun B, He H, Wang Z, et al. Emergent severe acute respiratory distress syndrome caused by adenovirus type 55 in immunocompetent adults in 2013: a prospective observational study. Crit Care 2014;18:456. 
14. Gao HW, Wei MT, Fan HJ, et al. Dynamic changes in clinical characteristics during an outbreak of human adenovirus serotype 55 in China. Disaster Med Public Health Prep 2018;12:464-469.

15. Lafolie J, Mirand A, Salmona M, et al. Severe pneumonia associated with adenovirus type 55 infection, France, 2014. Emerg Infect Dis 2016;22:2012-2014.

16. Salama M, Amitai Z, Amir N, et al. Outbreak of adenovirus type 55 infection in Israel. J Clin Virol 2016;78:31-35.

17. Wang W, Liu Y, Zhou Y, et al. Whole-genome analyses of human adenovirus type 55 emerged in Tibet, Sichuan and Yunnan in China, in 2016. PLoS One 2017;12:e0189625.

18. Zheng X, Rong X, Feng Y, et al. Seroprevalence of neutralizing antibodies against adenovirus type 14 and 55 in healthy adults in Southern China. Emerg Microbes Infect 2017;6:e43.

19. Kim SJ, Kim K, Park SB, Hong DJ, Jhun BW. Outcomes of early administration of cidofovir in non-immunocompromised patients with severe adenovirus pneumonia. PLoS One 2015;10:e0122642.

20. Yoo H, Gu SH, Jung J, et al. Febrile respiratory illness associated with human adenovirus type 55 in South Korea military, 2014-2016. Emerg Infect Dis 2017;23:1016-1020.

21. Hwang SM, Park DE, Yang YI, et al. Outbreak of febrile respiratory illness caused by adenovirus at a South Korean military training facility: clinical and radiological characteristics of adenovirus pneumonia. Jpn J Infect Dis 2013;66:359-365.

22. Park JY, Kim BJ, Lee EJ, et al. Clinical features and courses of adenovirus pneumonia in healthy young adults during an outbreak among Korean military personnel. PLoS One 2017;12:e0170592.

23. Heo JY, Noh JY, Jeong HW, et al. Molecular epidemiology of human adenovirus-associated febrile respiratory illness in soldiers, South Korea. Emerg Infect Dis 2018;24:1221-1227.

24. Kim HS, Ko RE, Ji M, Lee JH, Lee CS, Lee H. The usefulness of hand washing during field training to prevent acute respiratory illness in a military training facility. Medicine (Baltimore) 2018;97:e11594.

25. Heo JY, Lee JE, Kim HK, Choe KW. Acute lower respiratory tract infections in soldiers, South Korea, April 2011-March 2012. Emerg Infect Dis 2014;20:875-877.

26. Jeon K, Kang CI, Yoon CH, et al. High isolation rate of adenovirus serotype 7 from South Korean military recruits with mild acute respiratory disease. Eur J Clin Microbiol Infect Dis 2007;26:481-483.

27. Lee J, Choi EH, Lee HJ. Clinical severity of respiratory adenoviral infection by serotypes in Korean children over 17 consecutive years (1991-2007). J Clin Virol 2010;49:115-120.

28. Heo JY, Kim HK, Cha YJ, Lee JE, Shim YS, Choe KW. A clinical features of severe adenovirus pneumonia among members of the Korea military: a case series. Infect Chemother 2012;44:372-376.

29. Chen WW, Nie WM, Xu W, et al. Cross-sectional study of the relationship of peripheral blood cell profiles with severity of infection by adenovirus type 55 . BMC Infect Dis 2014;14:147.

30. Gu L, Liu Z, Li X, et al. Severe community-acquired pneumonia caused by adenovirus type 11 in immunocompetent adults in Beijing. J Clin Virol 2012;54:295-301.

31. Ko JH, Park GE, Lee JY, et al. Predictive factors for pneumonia development and progression to respiratory failure in MERS-CoV infected patients. J Infect 2016;73:468475 .

32. Park GE, Ko JH, Peck KR, et al. Control of an outbreak of Middle East respiratory syndrome in a tertiary hospital in Korea. Ann Intern Med 2016;165:87-93.

33. Choi WS, Kang CI, Kim Y, et al. Clinical presentation and outcomes of Middle East respiratory syndrome in the Republic of Korea. Infect Chemother 2016;48:118-126.

34. Barker JH, Luby JP, Sean Dalley A, Bartek WM, Burns DK, Erdman DD. Fatal type 3 adenoviral pneumonia in immunocompetent adult identical twins. Clin Infect Dis 2003;37:e142-e146.

35. Hoke CH Jr, Snyder CE Jr. History of the restoration of adenovirus type 4 and type 7 vaccine, live oral (adenovirus vaccine) in the context of the department of defense acquisition system. Vaccine 2013;31:1623-1632.

36. Liu T, Zhou Z, Tian X, et al. A recombinant trivalent vaccine candidate against human adenovirus types 3, 7, and 55. Vaccine 2018;36:2199-2206.

37. Feng Y, Sun X, Ye X, et al. Hexon and fiber of adenovirus type 14 and 55 are major targets of neutralizing antibody but only fiber-specific antibody contributes to cross-neutralizing activity. Virology 2018;518:272-283.

38. Tian X, Jiang Z, Fan Y, et al. A tetravalent vaccine comprising hexon-chimeric adenoviruses elicits balanced protective immunity against human adenovirus types 3, 7, 14 and 55. Antiviral Res 2018;154:17-25. 


\section{SUPPLEMENTARY MATERIAL}

\section{Laboratory tests}

During the study period, polymerase chain reaction (PCR) analyses of eight respiratory viruses (RVs) were performed at three clinical laboratories depending on period and hospital. Specimens collected from Hospitals A-I, K, and L-N for the entire study period, Hospital J from January 2013 to April 2014, and Hospital L from January 2013 to April 2016 were tested at Green Cross Co. (Yongin, Korea). Nucleic acid extraction was performed using NucliSENS easyMAG (bioMerieux, Marcy l'Etoile, France), and multiplex PCR was performed using Seeplex RV12 (Seegene, Seoul, Korea) as described previously $[21,25]$. Hospital J performed RV-PCR at the in-hospital clinical laboratory in May 2014. Nucleic acid extraction was performed using the MagNA Pure LC Nucleic Acid isolation kit I (Roche Diagnostics, Mannheim, Germany), and multiplex PCR was performed using the Real-Q RV Detection kit (BioSewoom, Seoul, Korea) [19]. Hospital L performed RV-PCR at the in-hospital laboratory from May 2016, using the TANBead Viral Auto Plate system (Taiwan Advanced Nanotech Inc., Taoyuan City, Taiwan) for nucleic acid extraction and the AdvanSure RV real-time PCR Kit (LG Life Sciences, Seoul, Korea) for multiplex PCR, as described previously [22].

\section{Epidemiologic patterns of the seven RVs other than human adenovirus}

Influenza virus (IFV) in the military exhibited almost the same seasonal variation as in civilians from 2013, even though the overall detection proportion was significantly lower. However, in the 2013 to 2014 period, the peak detection proportion of IFV in the military (61.6\%) was similar to that of civilians (68.3\%), implying an IFV outbreak in the military. Human parainfluenza virus (HPIV) was detected in the military beginning in the spring and ending in the summer, earlier than for civilians. The overall detection proportion of HPIV in the military was lower than for civilians $(p<0.001$ from the 2013 to 2014 period). HRSV in the military began to increase in the winter, later than for civilians, and ended in the spring. Seasonal variation (late fall to spring) and detection of human coronavirus in the military were very similar to those observed for civilians starting in 2015. Human rhinovirus (HRV) in the military exhibited similar seasonality to civilians (spring to early winter), but detection proportions increased steadily from the 2013 to 2014 period (from $2.5 \%$ to $17.4 \%, p=0.001$ by linear regression). Detection proportion of HRV during the nadir of the winter season in the military also increased. Although human bocavirus (HBoV) cases were observed during the spring to summer in civilians, few cases of $\mathrm{HBoV}$ were detected in the military. Human metapneumovirus exhibited similar seasonality in the military as in civilians, increasing from late winter to spring. 
Heo J, et al. Comorbidities among cancer survivors

Supplementary Table 1. Geographic location and size of the 14 included military hospitals

\begin{tabular}{llc}
\hline Hospital & \multicolumn{1}{c}{ Geographic region } & No. of beds \\
\hline A & Goyang City, Gyeonggi Province & 250 \\
B & Yangju City, Gyeonggi Province & 500 \\
C & Pocheon City, Gyeonggi Province & 250 \\
D & Chuncheon City, Gangwon Province & 230 \\
E & Hongcheon City, Gangwon Province & 230 \\
F & Guri City, Gyeonggi Province & 500 \\
G & Gangneung City, Gangwon Province & 180 \\
H & Seoul Metropolitan City & 30 \\
I & Wonju City, Gangwon Province & 20 \\
J & Seongnam City, Gyeonggi Province & 660 \\
K & Gyeongsan City, North Gyeongsang Province & 500 \\
L & Daejeon Metropolitan City & 600 \\
M & Busan Metropolitan City & 260 \\
N & Hampyeong County, South Jeolla Province & 500 \\
Total & All 14 military hospitals affiliated to AFMC & 4,710 \\
\hline AFMC, Arm & \\
\hline
\end{tabular}

AFMC, Armed Forces Medical Command. 
Supplementary Table 2. Comparison of positive percentage of human adenovirus in the periods from winter to spring and from summer to fall

\begin{tabular}{|c|c|c|c|c|c|c|}
\hline \multirow{2}{*}{ Period } & \multicolumn{2}{|c|}{ Winter to spring } & \multicolumn{2}{|c|}{ Summer to fall } & \multirow{2}{*}{$\begin{array}{c}p \text { value } \\
\text { (average comparison) }\end{array}$} & \multirow{2}{*}{ Peak/Nadir ratio } \\
\hline & Average & Peak & Average & Nadir & & \\
\hline $2014-2015$ & $58.3 \pm 12.93$ & 75.0 & $22.8 \pm 8.48$ & $15 \cdot 3$ & $<0.001$ & 4.90 \\
\hline $2015-2016$ & $40.5 \pm 6.04$ & $47 \cdot 7$ & $25 \cdot 2 \pm 5 \cdot 53$ & 17.1 & 0.001 & 2.79 \\
\hline $2016-2017$ & $61.3 \pm 13.06$ & 73.8 & $54.4 \pm 7.77$ & 41.5 & 0.295 & 1.78 \\
\hline
\end{tabular}

Values are presented as mean \pm standard deviation. 
Heo J, et al. Comorbidities among cancer survivors

Supplementary Table $3 \cdot p$ values for comparison of RV detection proportions between military and civilian hospitals

\begin{tabular}{|c|c|c|c|c|c|c|}
\hline \multirow[b]{2}{*}{ RVs } & \multicolumn{6}{|c|}{$p$ values for comparisons of military and civilian hospitals } \\
\hline & $\begin{array}{c}\text { January 2013- } \\
\text { May } 2013\end{array}$ & $\begin{array}{c}\text { June 2013- } \\
\text { May } 2014\end{array}$ & $\begin{array}{c}\text { June } 2014- \\
\text { May } 2015\end{array}$ & $\begin{array}{c}\text { June } 2015- \\
\text { May } 2016\end{array}$ & $\begin{array}{c}\text { June 2016- } \\
\text { May } 2017\end{array}$ & $\begin{array}{c}\text { June 2017- } \\
\text { May } 2018\end{array}$ \\
\hline HAdV & $<0.001$ & $<0.001$ & $<0.001$ & $<0.001$ & $<0.001$ & $<0.001$ \\
\hline IFV & $<0.001$ & $<0.001$ & $<0.001$ & $<0.001$ & $<0.001$ & $<0.001$ \\
\hline HPIV & 0.444 & $<0.001$ & $<0.001$ & $<0.001$ & $<0.001$ & $<0.001$ \\
\hline HRSV & 0.045 & 0.093 & $<0.001$ & 0.017 & $<0.001$ & $<0.001$ \\
\hline $\mathrm{HCoV}$ & 0.005 & 0.017 & $<0.001$ & 0.819 & $<0.001$ & 0.968 \\
\hline HRV & 0.340 & $<0.001$ & $<0.001$ & $<0.001$ & $<0.001$ & 0.040 \\
\hline $\mathrm{HBoV}$ & 0.771 & $<0.001$ & $<0.001$ & $<0.001$ & $<0.001$ & $<0.001$ \\
\hline HMPV & 0.406 & 0.083 & $<0.001$ & 0.206 & $<0.001$ & $<0.001$ \\
\hline Positive, total & 0.350 & 0.038 & $<0.001$ & 0.392 & $<0.001$ & $<0.001$ \\
\hline
\end{tabular}

RV, respiratory virus; HAdV, human adenovirus; IFV, influenza virus; HPIV, human parainfluenza virus; HRSV, human respiratory syncytial virus; $\mathrm{HCoV}$, human coronavirus; HRV, human rhinovirus; $\mathrm{HBoV}$, human bocavirus; HMPV, human metapneumovirus. 
Supplementary Table 4. Pneumonia surveillance results for 14 military hospitals and RV surveillance results for new recruits at the time of entrance

\begin{tabular}{|c|c|c|c|c|c|c|}
\hline Variable & $\begin{array}{l}\text { January } 2013- \\
\text { May } 2013\end{array}$ & $\begin{array}{l}\text { June 2013- } \\
\text { May } 2014\end{array}$ & $\begin{array}{l}\text { June 2014- } \\
\text { May } 2015\end{array}$ & $\begin{array}{l}\text { June } 2015- \\
\text { May } 2016\end{array}$ & $\begin{array}{l}\text { June 2016- } \\
\text { May } 2017\end{array}$ & $\begin{array}{l}\text { June 2017- } \\
\text { May } 2018\end{array}$ \\
\hline Pneumonia patients & 360 & 673 & 2,922 & 3,474 & 4,887 & 3,187 \\
\hline $\begin{array}{l}\text { HAdV detection in new recruits } \\
\text { at entrance }\end{array}$ & NA & NA & NA & 55 & 42 & 8 \\
\hline $\begin{array}{l}\text { RV-PCR tests in new recruits at } \\
\text { entrance }\end{array}$ & NA & NA & NA & 2,973 & 2,015 & 992 \\
\hline $\begin{array}{l}\text { HAdV positive percentage among } \\
\text { new recruits at entrance }\end{array}$ & NA & NA & NA & $1.9 \%$ & $2.1 \%$ & $0.8 \%$ \\
\hline
\end{tabular}

The number of patients with pneumonia from the 14 military hospitals was reported to the Korean Armed Forces Medical Command according to the International Classification of Disease and Related Health Problems, 1oth revision, codes J12-J18. RV surveillance in new recruits was performed by the Korea Army Training Center from August 2015, for symptomatic new recruits within 1 week of entrance (before initiation of major training programs). RV, respiratory virus; HAdV, human adenovirus; PCR, polymerase chain reaction. 
Heo J, et al. Comorbidities among cancer survivors

Supplementary Table 5. Severe HAdV-ARIs in the Korean military

\begin{tabular}{|c|c|c|c|c|c|c|c|}
\hline Variable & $\begin{array}{l}\text { January 2013- } \\
\text { May } 2013\end{array}$ & $\begin{array}{l}\text { June 2013- } \\
\text { May } 2014\end{array}$ & $\begin{array}{l}\text { June 2014- } \\
\text { May 2015 }\end{array}$ & $\begin{array}{l}\text { June } 2015- \\
\text { May } 2016\end{array}$ & $\begin{array}{l}\text { June 2016- } \\
\text { May } 2017\end{array}$ & $\begin{array}{l}\text { June 2017- } \\
\text { May } 2018\end{array}$ & Total \\
\hline HAdV detection & 43 & 109 & 1,286 & 1,037 & 2,480 & 1,691 & 6,646 \\
\hline Cidofovir treatment & 0 & 5 & 29 & 19 & 28 & 25 & 106 \\
\hline Cidofovir treatment, \% & 0.0 & 4.6 & 2.3 & 1.8 & 1.1 & 1.5 & 1.6 \\
\hline $\mathrm{RF}$ requiring $\mathrm{MV}$ & o & 2 & 8 & 5 & 9 & 8 & 32 \\
\hline $\mathrm{RF}, \%$ & 0.0 & 1.8 & 0.6 & 0.5 & 0.4 & 0.5 & 0.5 \\
\hline ECMO support & o & o & 4 & o & 4 & 7 & 15 \\
\hline ECMO support, \% & 0.0 & 0.0 & 0.3 & 0.0 & 0.2 & 0.4 & 0.2 \\
\hline Death & o & o & 2 & 0 & 2 & 1 & 5 \\
\hline Fatality rate, \% & 0.000 & 0.000 & 0.156 & 0.000 & 0.081 & 0.059 & 0.075 \\
\hline $\begin{array}{l}\text { Fatality rate in patients } \\
\text { with RF, \% }\end{array}$ & 0.0 & 0.0 & 25.0 & 0.0 & 22.2 & 12.5 & 15.6 \\
\hline
\end{tabular}

HAdV, human adenovirus; ARI, acute respiratory illness; RF, respiratory failure; MV, mechanical ventilation; ECMO, extracorporeal membrane oxygenation. 
A
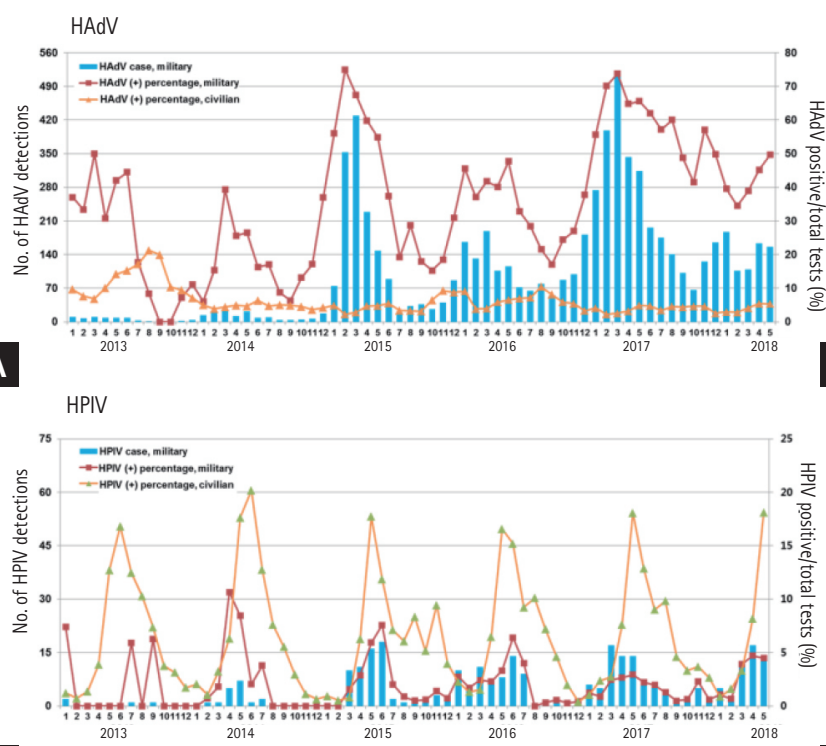

C

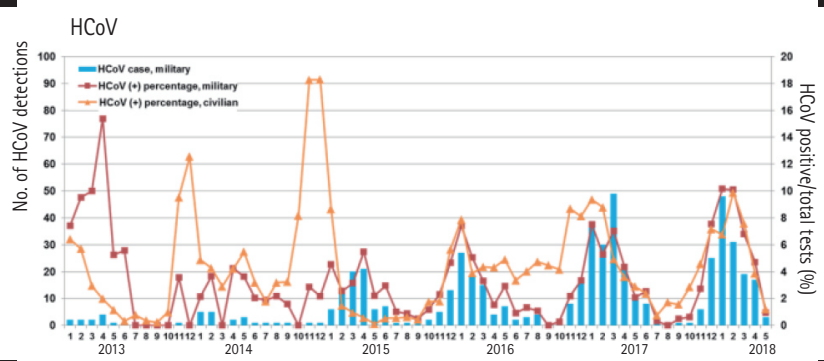

E

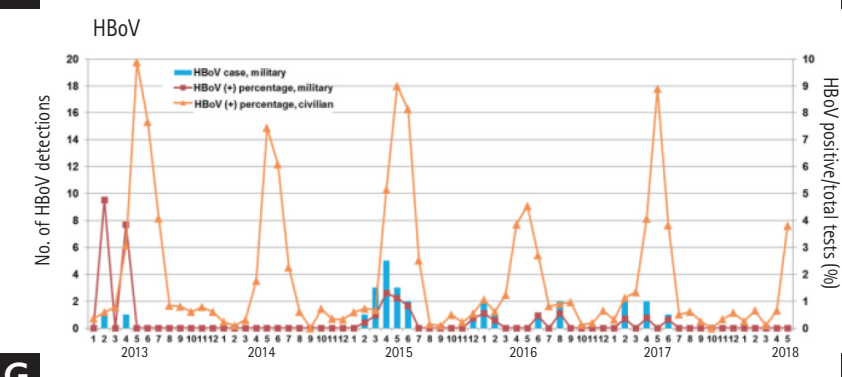

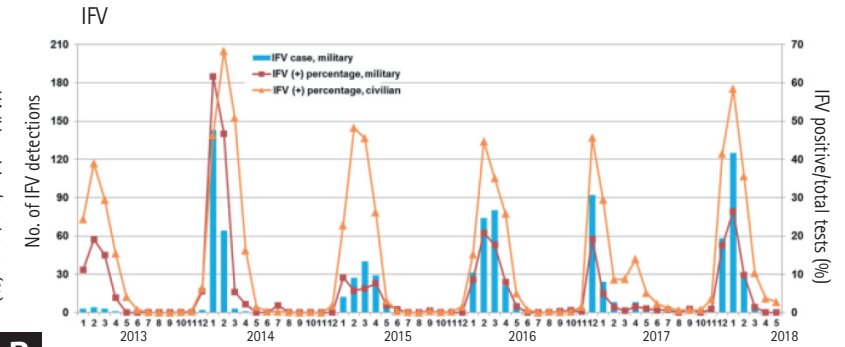

B

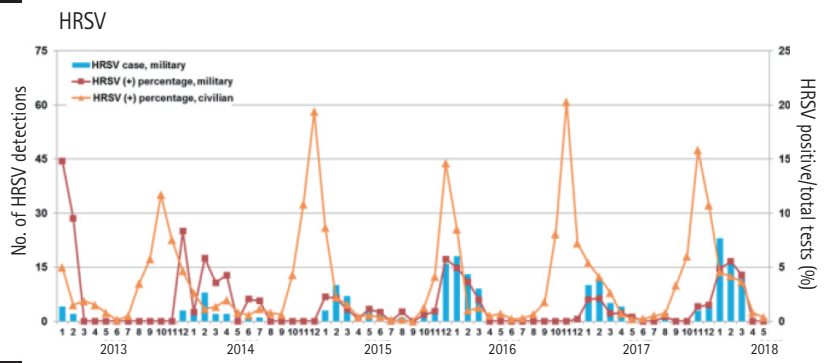

D

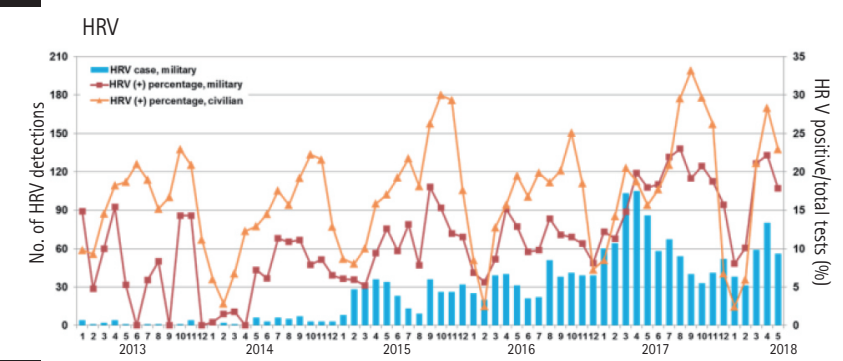

$\mathbf{F}$

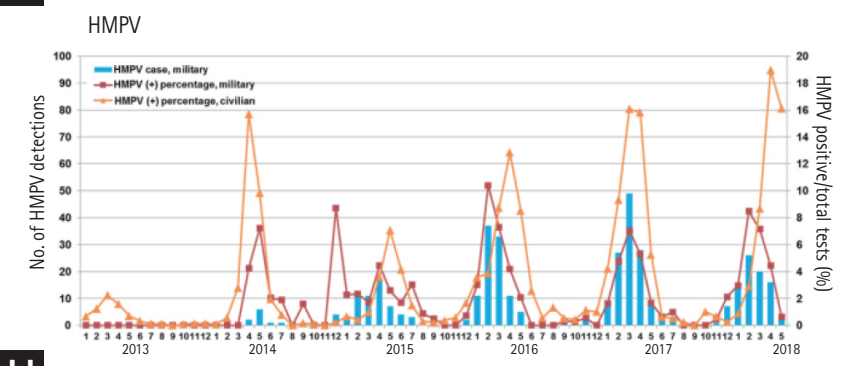

Supplementary Figure 1. Epidemiologic trends of eight respiratory viruses (RVs) in the Korean military in comparison with the civilian population, with the scale adjusted for each RV. RV-polymerase chain reaction results for 14 military hospitals from January 2013 to May 2018 are presented with nationwide surveillance data from civilian hospitals. (A) Human adenovirus (HAdV), (B) influenza virus (IFV), (C) human parainfluenza virus (HPIV), (D) human respiratory syncytial virus (HRSV), (E) human coronavirus (HCoV), (F) human rhinovirus (HRV), (G) human bocavirus (HBoV), and $(\mathrm{H})$ human metapneumovirus (HMPV). 